\title{
Endoscopia lumbar póstero lateral con sistema YESS - reporte preliminar
}

\author{
Endoscopia lombar póstero-lateral com sistema YESS - \\ resultados preliminares
}

\section{Lumbar disc herniation treated with post lateral lumbar endoscopy by YESS system - preliminary results}

\author{
Carlos Montes García'
}

\section{RESUMEN}

Introducción: en los pacientes con diagnóstico de hernia de disco lumbar, los cuales son candidatos a tratamiento quirúrgico, la disectomía posterior abierta ha sido por muchos anos el estándar de oro. Sin embargo, las complicaciones de este procedimiento, en el que se invade el canal medular, han hecho que se busquen otros métodos menos invasivos. Uno de estos procedimientos es la disectomía endoscópica lateral con el método YESS (Young Endoscopic Spine System). Objetivo: revisar los resultados por dos años de cien pacientes diagnosticados con hernia de disco lumbar, tratados con endoscopia lateral con sistema YESS. Métodos: se estudiaron cien pacientes con diagnóstico de hernia de disco lumbar comprobada con resonancia magnética y discografía evocativa positiva. Todos los pacientes nunca habían hecho un tratamiento quirúrgico y se les dio seguimiento durante dos años. Resultados: los resultados se clasificaron en buenos, regulares, y malos. Se encontraron buenos resultados en 82 pacientes, regulares en 10, y malos en 8. Conclusiones: los resultados del tratamiento de la hernia de disco lumbar con la endoscopia lateral y sistema YESS son, hasta el momen-

\section{RESUMO}

Introdução: nos pacientes com diagnóstico de hérnia de disco lombar, candidatos ao tratamento cirúrgico, a discectomia posterior aberta tem sido por muitos anos o padrão-ouro. Embora as complicações deste procedimento, no qual se invade o canal medular, têm levado a procurar outros métodos menos invasivos. Um destes procedimentos é a discectomia endoscópica lateral com o método YESS (Young Endoscopic Spine System). Objetivo: revisar os resultados por dois anos de cem pacientes com diagnóstico de hérnia de disco lombar tratados com endoscopia lateral, com sistema YESS. Métodos: foram estudados cem pacientes com diagnóstico de hérnia de disco lombar, comprovada com ressonância magnética e discografia evocativa positiva. Todos os pacientes nunca tinham passado por um tratamento cirúrgico e foi feito um seguimento por dois anos. Resultados: os resultados foram classificados em bons, regulares e ruins. Foram encontrados bons resultados em 82 pacientes, regulares em 10 e ruins em 8. Conclusões: os resultados do tratamento da hérnia de disco lombar, com a endoscopia lateral e com o sistema YESS são, até o momento, similares

\begin{abstract}
Introduction: in the patients with lumbar disc herniation, who are candidates to surgical procedures, the open diskectomy has been the gold standard for many years. Nevertheless, the complications of this procedure, in which the medullar canal is invaded, have caused other means to be developed. One of them is the YESS system (Young Endoscopic Spine System). Objective: to examine the results of one hundred patients with lumbar disc herniation, treated with the YESS system. Methods: it was studied one hundred patients with lumbar disc herniation, which was proved with magnetic resonance imaging and discography, and their results were positive. None of the patients had ever undergone a surgical procedure; therefore, they were tracked for two years. Results: results were classified into good, regular, and bad. In 82 patients good results were found, 10 were regular, and 8 were bad. Conclusions: the results of the treatment for a lumbar disc herniation with the YESS system are so far similar to open back surgery, even though the time of recovery, disability, and costs are
\end{abstract}

\footnotetext{
Trabajo hecho en lo Hospital Poliplaza Medica - Ciudad Juarez, Chihuahua, México. 
to, similares con el abordaje posterior abierto, aunque el tiempo de recuperación y capacidad son menores así también como el costo, ya que es un procedimiento ambulatorio.

DESCRIPTORES: Endoscopía/ métodos; Desplazamiento del disco intervertebral/cirugía; Vértebras lumbares/cirugía; Procedimientos quirúrgicos mínimamente invasivos; Discitis/microbiología; Discectomía /métodos àqueles com abordagem posterior aberta, mesmo que o tempo de recuperação e a capacidade sejam menores, assim como também o custo, pois é um procedimento ambulatório. lower because it is an ambulatory procedure.

\author{
DESCRITORES: Endoscopia/ \\ métodos; Deslocamento do \\ disco intervertebral/cirurgia; \\ Vértebras lombares/cirurgia; \\ Procedimentos cirúrgicos \\ minimamente invasivos; \\ Discite/microbiologia; \\ Discotomia/métodos
}

\author{
KEYWORDS: Endoscopy/ \\ methods; Intervertebral \\ disk displacement/surgery; \\ Lumbar vertebrae/surgery; \\ Surgical procedures, \\ minimally invasive; Discitis/ \\ microbiology; Diskectomy/ \\ methods
}

\section{INTRODUCCIÓN}

Los pacientes con lumbociática causada por hernia de disco intervertebral, que comprime la raíz nerviosa y en aquellos que la respuesta al tratamiento médico y fisioterapia; las inyecciones epidurales con esteroides han fracasado y la sintomatología persiste por más de 12 semanas, son candidatos al tratamiento quirúrgico ${ }^{1}$. El tiempo de espera es justificado pues un $75 \%$ se recuperan y esto no afecta el resultado del tratamiento quirúrgico ${ }^{2}$. El abordaje posterior abierto es la técnica quirúrgica considerada el estándar de oro para remover el disco herniado y descomprimir el canal espinal ${ }^{3-5}$. Sin embargo, la retracción muscular; la resección parcial de lámina y de faceta articular; la retracción de la duramadre y de la raíz nerviosa; y el uso de anestesia general llevan riesgos trans y postoperatorios. Estos pueden causar fibrosis peridural, dolor quirúrgico crónico e inestabilidad vertebral, así como lesiones durales con fístula de líquido céfalorraquídeo. Complicaciones que pueden generar procesos médico legales que representan una verdadera pesadilla tanto para el paciente como para el cirujano ${ }^{6}$.

El desgarro dural es causa común de demanda ${ }^{7,8}$, y en algunos casos se asocia con síndrome de cauda equina, la cual puede dejar severas secuelas neurológicas 9 .

Es por eso que durante los últimos 40 años ha habido un importante desarrollo en las técnicas de mínima invasión para la descompresión discal, y para el alivio tanto del dolor lumbar discógeno crónico como el dolor radicular, evitando los riesgos de la cirugía abierta ${ }^{10}$. La meta de estos procedimientos es tratar al paciente usando métodos que reduzcan el trauma operativo y que los pacientes puedan regresar rápidamente a sus actividades diarias con un mínimo dolor ${ }^{11}$.

El incremento de los procedimientos de mínima invasión ha conducido a la creación de un nuevo campo de la cirugía espinal. Han aparecido instrumentos como el endoscopio vertebral con canal de trabajo, la radiofrecuencia bipolar para hemostasia y contracción térmica del colágeno del anillo fibroso y ablación térmica de los nociceptores.

El concepto de la descompresión indirecta del canal espinal por una vía póstero-lateral extra canal fue introducido por Kambin y Gellmann en 1983, usando una cánula de Craig ${ }^{12}$. Kambin describió una zona de trabajo, hoy conocida como triángulo de Kambin ${ }^{13,14}$ (Figura 1), que permite el acceso seguro para los instrumentos y que es constituida de la siguiente manera: su base es la plataforma proximal del cuerpo de la vertebra infrayacente, su altura el proceso articular proximal y la hipotenusa es la raíz nerviosa emergente $^{15}$.

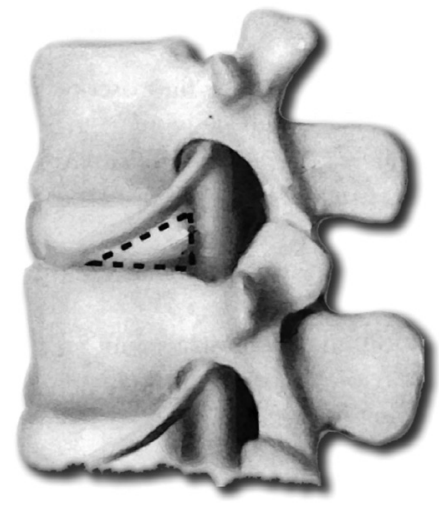

Figura 1

Triángulo de Kambin.

Yeung y Tsou introdujeron el endoscopio con canal de trabajo integrado (Figura 2) y siguiendo los principios pioneros de Kambin, desarrolló la técnica quirúrgica conocida como YESS; con la cual se puede abordar tanto la patología discal como la foraminal ${ }^{16}$. Los resultados de diversos estudios son equiparables con los de la cirugía abierta, la menor morbilidad y más rápida recuperación nos hacen preferirla. 


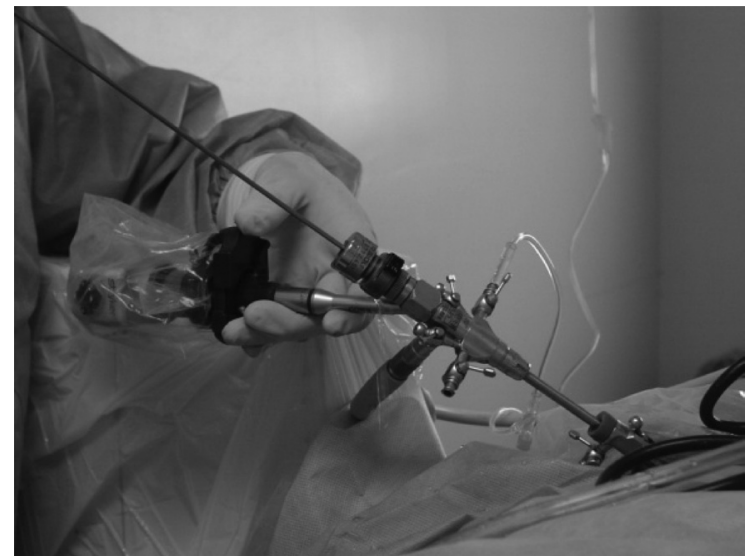

Figura 2

Endoscopio de Yang.

\section{OBJETIVO}

Revisar resultados a dos años de seguimiento de 100 pacientes con diagnóstico de hernia de disco lumbar, tratados con endoscopia póstero-lateral con sistema Yess.

\section{MÉTODOS}

Se estudiaron cien pacientes con diagnóstico de hernia de disco lumbar, comprobada con resonancia magnética y discografía evocativa positivas. Las hernias podían ser únicas o múltiples, de localización póstero-lateral, en grado de protrusión o extrusión. (Figura 3), con una evolución clínica mayor de 12 semanas y que no respondieron al tratamiento conservador.

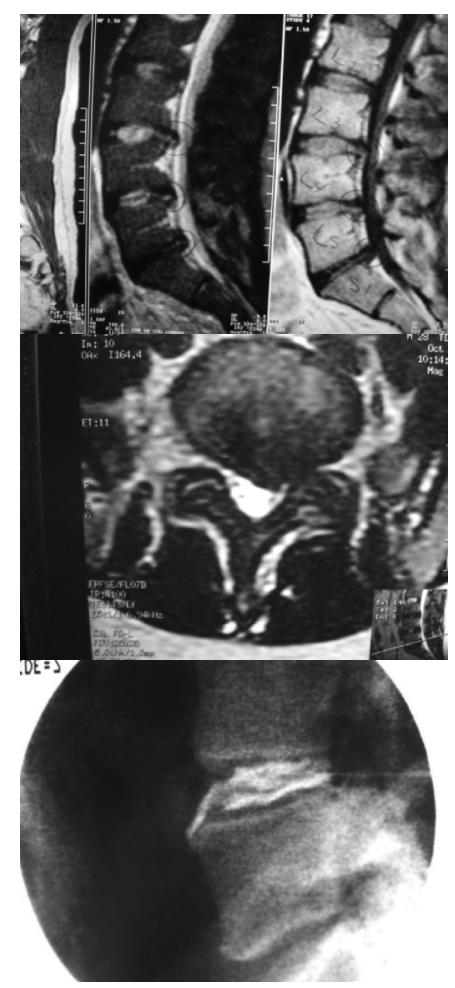

Figura 3

Resonancia magnética y discografía positiva.
Se excluyeron los casos de espondilolistesis, canal lumbar estrecho, pacientes ya operados con hernias secuestradas o posteriores, con resonancia magnética (RM) positiva pero discografía negativa ${ }^{17}$.

De los 100 pacientes, 65 fueron evaluados en forma personal, 25 por vía telefónica y 10 respondieron a un formulario que se les envió por correo.

Los resultados se clasificaron en tres categorías:

- buenos - remisión completa del dolor, regreso sin limitaciones a sus actividades, satisfacción personal;

- regulares - remisión parcial del dolor, regreso a sus actividades con algunas limitaciones pero aún satisfecho;

- malos - dolor residual importante, parestesias, no pudieron regresar a su trabajo o lo hicieron con muchas limitaciones, obviamente muy insatisfechos.

\section{EL PROCEDIMIENTO}

Con el paciente en decúbito ventral, bajo sedación y anestesia local. Asistidos con el fluoroscopio, se trazan sobre la piel tres líneas cutáneas cuya convergencia en un punto nos indica el sitio de entrada de la aguja iniciadora. Las primeras dos son en posición AP; una vertical que sigue el trayecto de las apófisis espinosas y otra horizontal que pasa por el espacio intervertebral y que en su intersección con la primera nos indica el centro del disco, finalmente en posición lateral se traza una tercera línea que indica la inclinación cefalocaudal del disco (Figura 4). La convergencia de estas líneas marca la puerta de entrada.

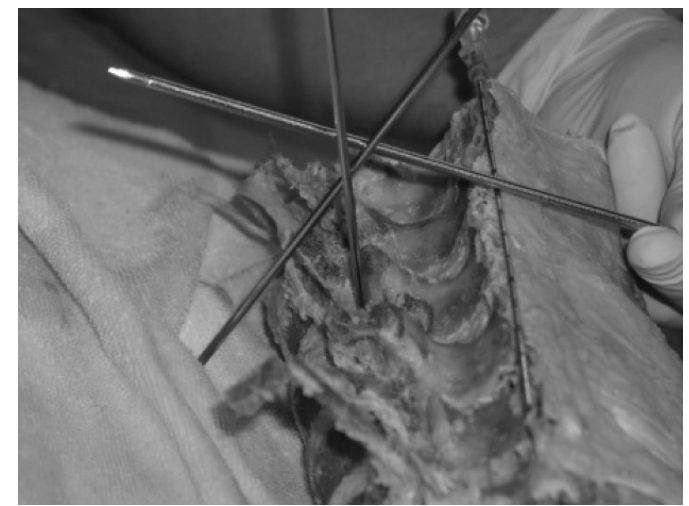

Figura 4

Localización del triángulo de Kambin en cadáver.

Después de asepsia con Durapred ${ }^{\circledR}$ y colocación de campos estériles se aplican $5 \mathrm{cc}$ de lidocaína al $1 \%$ en el sitio de entrada. Se introduce una aguja de $17 \mathrm{~cm}$ de largo, número 18 con una inclinación entre 35 y 45 grados dependiendo del grosor del paciente y la cual se dirige al triángulo de Kambin hasta llegar al núcleo pulposo. Se inyecta una mezcla de medio de contraste tipo Optiray ${ }^{\circledR}$, agua bidestilada e índigo carmín (que tiene afinidad por el núcleo pulposo y lo tiñe de azul).

Si la aplicación del medio resulta indolora se encuentra resistencia pasando no más de $1.5 \mathrm{cc}$ y se distribuye en forma oval, la discografía se considera negativa y se termina el procedimiento. 
Pero si la aplicación del medio reproduce el dolor del paciente, hay poca resistencia a la inyección, admite más de $3 \mathrm{cc}$ y su distribución es irregular ascendiendo en algunas ocasiones por el canal medular. La discografía se considera positiva y se continúa con el procedimiento endoscópico (Figura 3)

\section{RESULTADOS}

Participaron 44 mujeres y 56 hombres entre 30 y 50 años de edad, de ocupaciones diversas como enfermeras, secretarias, trabajadores del campo y de la industria. La localización de la hernia fue: 70 casos (L5S1), 65 (L4L5), 10 (L3L4) y 1 caso (L2L3). Esto nos da un total de 146 hernias, de las cuales, 62 únicas, 30 hernias a 2 niveles y 8 hernias en 3 niveles. Con lateralización izquierda en 80 pacientes, derechas en 50 y medial en 16 . El $96 \%$ de los casos requirió incapacidad entre dos y cuatro semanas.

La evaluación final de los resultados fue: para buenos resultados con 82 pacientes, regulares con 10 y malos con 8 , siendo que cinco presentaron ciática residual, seis lumbalgia residual y dos parestesias en miembros pélvicos. Cinco pacientes desarrollaron discitis bacteriana (Figura 5), los cuales estuvieron cuatro semanas de hospitalización con antibióticos intravenosos y limpieza quirúrgica del área. Todos los pacientes infectados fueron hombres y todos los casos se resolvieron satisfactoriamente. Los pacientes con ciática residual y parestesias fue cuando la hernia estaba localizada entre L5 y S1. Los seis pacientes con lumbalgia residual se observaron en pacientes en que el disco había perdido más de una tercera parte de su altura.

\section{DISCUSIÓN}

Hasta el momento los resultados de la endoscopia pósterolateral son comparables con los del abordaje posterior abierto. Evita desgarros del saco dural, complicaciones frecuentes y causa de demanda medico legal en estos pacientes.

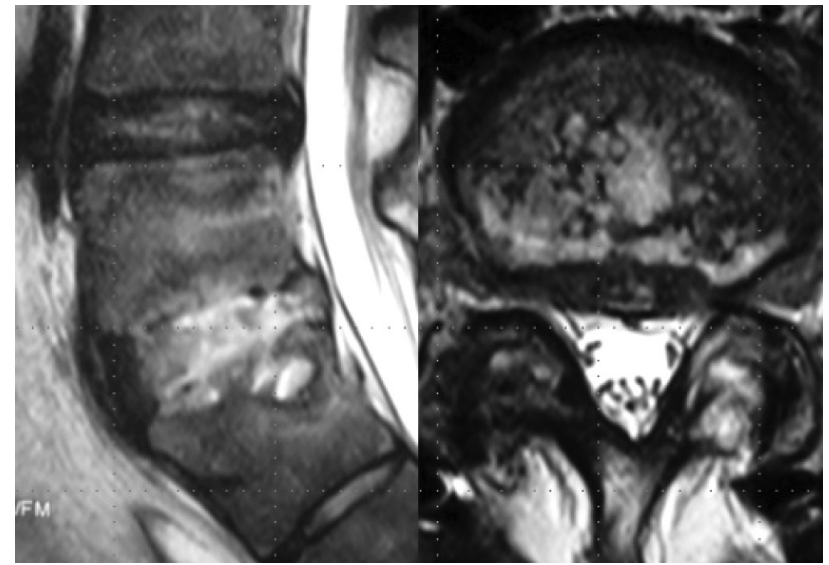

Figura 5

Discitis L5-S7.

El tiempo de recuperación y la incapacidad laboral son menores, el protocolo es de ambulatorio, disminuyendo el costo hospitalario.

En esta serie en efecto no hubo desgarros del saco dural, la recuperación de los pacientes fue rápida y el periodo de incapacidad mínimo. Cinco pacientes presentaron discitis bacteriana grave e incapacitante, potencialmente generadoras de situaciones médico legales. Se resolvieron usando antibióticos parenterales a través de catéter central durante diversas semanas con reposo en cama e inmovilización con órtesis. Tratamiento prolongado y costoso, con recuperación lenta, acompañada de dolor intenso que obliga el paciente a permanecer varios meses fuera de su trabajo. Esta complicación cuando se presenta es suficiente para minimizar todas las ventajas de la endoscopia. En los casos en que se presentó, hubo el antecedente de haber esterilizado el instrumental con químicos como el Cidex ${ }^{\circledR}$ o el Microcin ${ }^{\circledR}$, por lo cual su utilización es desaprobada.

\section{REFERENCIAS}

1. Atlas SJ, Keller RB, Chang Y, Deyo RA, Singer DE. Surgical and nonsurgical management of sciatica secondary to lumbar disc herniation: five-year outcomes from the Maine Lumbar Spine Study. Spine. 2001;26(10):1179-87.

2. Bell GR, Rothman RH. The conservative treatment of sciatica. Spine. 1984;9(1):54-6.

3. Fager CA. Surgery of the lumbar spine. In: Symon L, Thomas DGT. Clark K, editors. Rob \& Smith's operative surgery. 4th; ed. London Butterworths; 1989. p. 405-6.
4. Barrios C, Ahmed M, Arrótegui J, Björnsson A, Gillström P. Microsurgery versus standard removal of the herniated lumbar disc. A 3-year comparison in 150 cases. Acta Orthop Scand. 1990;61(5):399-403.

5. Kho HC, Steudel WI. [Comparison of the microsurgical lumbar intervertebral disk operation with the conventional technic in free sequestered intervertebral disk prolapse. A retrospective study based on 267 cases]. Neurochirurgia (Stuttg). 1986;29(5):181-5.
6. Horwitz NH, Rizzoli HV. Herniated intervertebral discs and spinal stenosis. In: Horwitz NH, Rozzoli HV, editors. Postoperative complications of extracranial neurological surgery. Beltimore: Williams \& Wilkins, 1987. p. 30-72.

7. Jones AA, Stambough JL, Balderston RA, Rothman RH, Booth RE Jr. Longterm results of lumbar spine surgery complicated by unintended incidental durotomy. Spine. 1989;14(4):443-6.

8. Stolke D, Sollmann WP, Seifert V. Intra- and postoperative complications in lumbar disc surgery. Spine. 1989;14(1):56-9. 
9. Spector LR, Madigan L, Rhyne A, Darden B 2nd, Kim D. Cauda equina syndrome. J Am Acad Orthop Surg. 2008;16(8):471-9.

10.Mayer HM. Spine update.

Percutaneous lumbar disc surgery. Spine. 1994;19(23):2719-23.

11.Chen Y, Derby R, Lee S.

Descompresión discal percutánea en el tratamiento del dolor discal crónico. Orthop Clin North Am (ed. esp.). 2004;4:67-76.

12.Kambin P, Gellman H. Percutaneous lateral discectomy of the lumbar spine. A preliminary report. Clin Orthop. 1983;(174):127-32.
13. Kambin P. Arthroscopic microdisectomy. Arthroscopy. 1992;8(3):287-95.

14.Kambin P, Shofter JL. Percutaneous lumbar disectomy. Review of 100 patients and current practice. Clin Orthop. 1989;(238):145-54.

15.Yeung AT. Minimally invasive disc surgery with the Yeung Endoscopic Spine System (Y.E.S.S). Surg Technol Int. 1999;8(1):1-11.

16. Yeung AT, Tsou PM. Posterolateral endoscopic excision for lumbar disc herniation: Surgical technique, outcome and complications in 307 consecutive cases. Spine. 2002;27(7):722-31
17.Montes GC, Nava GLF. Discografía evocativa lumbar. Acta Ortop Mex. 2007;21(2):85-9.

\section{Correspondência}

Carlos Montes García

Avenida Profesor Pedro Rosales de León, 7.510

C.P. 32500

Ciudad Juárez Chihuahua, México.

Teléfono: 52 656-6236-911

E-mail: carlo104@prodigy,net.mx 